Conclusions In conclusion, we notice a good level of quality concerning prescriptions in this geriatric unit where software-assisted prescribing with pharmaceutical analysis has been effective since 2009. This software does not allow physicians to organise prescriptions by disease area. Concerning the patients' weight, senior clinicians will inform junior clinicians of its importance in the patients' file and prescription. Another evaluation will be scheduled to analyse the link between the number of drugs and the number of diseases. The final aim is to reduce the number of drugs in order to avoid drug-related adverse events.

No conflict of interest.

\section{GRP-068 EVALUATION OF SPECTRAL SPECIFICITY OF TAXANES FOR THE ON-LINE ANALYTICAL CONTROL OF HOSPITAL CHEMOTHERAPY PRODUCTION}

doi:10.1136/ejhpharm-2013-000276.068

G Sayet, L Havard, P Prognon, E Caudron. Hopital Européen Georges Pompidou, Pharmacy, Paris, France

Background On-line control of chemotherapy production is used for 27 molecules at European Georges Pompidou Hospital which corresponds to $70 \%$ of the production. Flow injection analysis (FIA) constitutes the optimal method under ultra-violet spectral data identification. If the spectra are similar, the retention time after chromatographic separation has to be used for identification. The FIA spectral differences of taxanes (docetaxel, paclitaxel, cabazitaxel) are too poor for identification.

Purpose The aim of this study was to develop an ultra-fast high performance liquid chromatographic technique for on-line analytical checking of taxane preparations.

Materials and Methods Docetaxel (Sanofi-Aventis), paclitaxel (Hospira) and cabazitaxel (Sanofi-Aventis) were prepared in sodium chloride $0.9 \%$ solution. Chromatography was performed using Prostar Varian chromatographic equipment with a Photodiode Array Detector. All the separation was done with a Polaris C18 precolumn $(3 \mu \mathrm{m}, 10 \mathrm{~mm} \times 2 \mathrm{~mm})$. The mobile phase was ultra-pure water/acetonitrile $(60-40 \mathrm{v} / \mathrm{v})$. Taxanes were eluted at the flow rate of $1.2 \mathrm{~mL} \cdot \mathrm{min}^{-1}$

Results Paclitaxel spectra obtained after chromatographic separation differ significantly from those of cabazitaxel and docetaxel, which are very similar. So the latter have to be identified by their retention time: $0.7 \mathrm{~min}$ for cabazitaxel and $0.4 \mathrm{~min}$ for docetaxel with a resolution of 1.7. Paclitaxel retention time was 0.39 with a resolution of 0.11 with docetaxel. The linear range corresponds to the therapeutic concentrations. The 3 methods were linear $(\mathrm{R}>0.995)$ with intra-day precision from $0.27 \%$ to $2.68 \%$ and inter-day precision from $0.95 \%$ to $3.7 \%$.

Conclusions Ultra-fast chromatographic separation methods have been successfully developed for the identification and quantification of 3 different taxane molecules. Less than $1 \mathrm{~min}$ is needed when spectral and retention data are combined as the main parameters.

No conflict of interest.

\section{GRP-069 EVALUATION OF THE CLINICAL IMPACT OF MEDICINES RECONCILIATION IN THE COMPIËGNE HOSPITAL CENTRE AFTER ONE YEAR OF EXPERIENCE}

doi:10.1136/ejhpharm-2013-000276.069

AC Desbuquois, F Lopes, C Aguerre, AM Liebbe. Hospital Center of Compiègne, Oise, Compiègne, France

Background The literature shows that there are errors in the drug treatment in $30 \%$ of patients at hospital admission. These medicines errors (MEs) may be continued throughout hospitalisation and can cause the patient adverse effects.

To reduce MEs and thus improve patient safety, the Compiègne Hospital Centre (HCC) has established a practise of medicines reconciliation (MR) since July 2011. Any unintentional discrepancies (UIDs) detected between the home treatment and the hospital treatment during MR are discussed and corrected with physicians to ensure continuity of the patient's medicines.

Purpose After one year of experience, the objective was to evaluate the clinical impact of our interventions on patient safety.

Materials and Methods Patients older than 65 years, hospitalised in Geriatrics and Cardiology after admission by the emergency department, were eligible for MR.

To evaluate the clinical impact of $M R$, we assessed the potential aftermath of uncorrected UIDs on patient safety. To do this, any UIDs detected and corrected were classified into two groups:

- those with a high potential clinical impact: potentially lifethreatening, that increase the length of hospitalisation and/ or decompensation/aggravation of an existing disease

- those with a low potential clinical impact.

Results 485 patients have benefited from MR, 30\% of whom had a $M E$ in their hospital prescription. Average age of patients: 84.6 years \pm 7.8 . Sex ratio M/F: 0.67.

259 UIDs were detected of which 101 (39\%) were classified as having a high potential clinical impact. This demonstrates the importance of MR for the safety of patients at their admission.

Conclusions After one year of MR in HCC, the results were positive.

The results on the clinical impact of our intervention were very encouraging and demonstrated the importance of continuing and developing medicines reconciliation. Our experience confirms the benefit of a pharmaceutical presence in the care units to improve patient safety.

No conflict of interest.

\section{GRP-070 EVALUATION OF THE EFFECT ON PATIENT SAFETY OF A NEW LABEL DESIGN FOR MEDICINAL PRODUCTS}

doi:10.1136/ejhpharm-2013-000276.070

${ }^{1} \mathrm{H}$ Fischer, ${ }^{1} \mathrm{MH}$ Clemmensen, ${ }^{1} \mathrm{~T}$ Kart, ${ }^{2} \mathrm{P}$ Dieckmann, ${ }^{2} \mathrm{P}$ Kunstek, ${ }^{3} \mathrm{~S}$ Schytte-Hansen, ${ }^{3}$ A Hellebek. 'Amgros, The Danish Research Unit for Hospital Pharmacy, Copenhagen OE, Denmark; 'Danish Institute for Medical Simulation (DIMS), Herlev Hospital University of Copenhagen, Herlev, Denmark; ${ }^{3}$ Regional Office for Patient Safety, Capital Region of Denmark, Hvidovre, Denmark

Background In Denmark some of the medicinal products for hospitals are produced by the hospital pharmacies and registered by Amgros (SAD products). In 2007 the Danish Society for Patient Safety, Amgros, and the private foundation TrygFonden organised a design competition with the purpose of improving patient safety in label design. The winner "Medilabel Safety System" was designed by e-Types and incorporated 9 design features. The new labels were implemented in 2008

Purpose To evaluate the effects of the new label design on patient safety.

Materials and Methods Reports of medication errors related to $\mathrm{SAD}$ products before and after the introduction of the new design (2007 and 2010) were compared. Medication errors were obtained from the Danish Patient Safety Database (DPSD).

In another study patient simulation and a sorting exercise were used to evaluate the effects of the new design. 11 physicians and 9 nurses participated.

Results In 2007 and 2010 a total of 6781 and 10188 medication errors were reported to DPSD. Of these, 85 (2007) and 80 (2010) dispensing errors could be related to misinterpretation of the SAD label. Thus, while no overall effect on the number of errors related 
to $\mathrm{SAD}$ products could be observed, the relative decrease could indicate a positive effect.

The simulation study indicated that specific design features such as yellow background colour, Tall Man lettering and consistent design improved safety in the medication process. However, the new label design is complex implying a potential for misinterpretation of the features if the users are not familiar with the design.

Conclusions The effect of the new design depends on several factors such as the user's knowledge of the design, the complexity of the design and the context of use. Errors related to misinterpretation of labels remains a problem and research into good label design remains a relevant topic.

No conflict of interest.

\section{GRP-071 EVALUATION OF THE PRESCRIPTION OF INTRAVENOUS NON-STEROIDAL ANTI-INFLAMMATORY DRUGS COMPARED TO THE RECOMMENDATIONS OF THE SUMMARY OF PRODUCT CHARACTERISTICS}

doi:10.1136/ejhpharm-2013-000276.071

C Salazar Saldebenito, MJ De Dios Garcia, M Alcalde Rodrigo, C Gonzalez-Guerrero, JB Montoro Ronsano, M Munné Garcia, I Cardona Pascual. Hospital Universitari Vall d'Hebron, Pharmacy, Barcelona, Spain

Background Acute renal failure is a side effect of NSAIDs.

Purpose To assess the appropriateness of the intravenous prescription of dexketoprofen according to the dosage specifications depending on renal function following the recommendations of the Summary of Product Characteristics.

Materials and Methods An observational, retrospective study that analysed dexketoprofen prescriptions in surgical patients admitted to a tertiary hospital from January-September 2011. The estimated glomerular filtration rate (eGF) was calculated by the CKD-EPI formula, of reference in the hospital.

The Summary of Product Characteristics advises using the following posology for dexketoprofen:

- $150 \mathrm{mg}$ maximum daily dose for a maximum duration of 48 hours.

- In patients with renal impairment:

- $\mathrm{GF}<50 \mathrm{~mL} / \mathrm{min}$ : administration contraindicated

- GF $50-80 \mathrm{~mL} / \mathrm{min}: 25 \mathrm{mg} / 12 \mathrm{~h}$. Maximum: $50 \mathrm{mg}$ daily.

- $\mathrm{GF}>80 \mathrm{~mL} / \mathrm{min}$ : No dosage adjustment required.

Results Prescriptions from 1946 patients were analysed. Of the patients, $54.3 \%$ were male and $45.7 \%$ female, with a mean age of 59.8 years $(17-103)$. The mean serum creatinine levels were $0.84 \mathrm{mg} / \mathrm{dL} \pm 0.43$ and the mean eGF from the CKD-EPI calculation was $83.05 \pm 26.17 \mathrm{~mL} / \mathrm{min} / 1.73 \mathrm{~m}^{2}$.

In $58 \%$ of the admissions the drug was not prescribed correctly. Of these:

- 270 patients were prescribed dexketoprofen when the eFG was less than $50 \mathrm{~mL} / \mathrm{min} / 1.73 \mathrm{~m}^{2}$;

- 550 of them had an unadjusted prescription with an eFG $50-80 \mathrm{~mL} / \mathrm{min} / 1.73 \mathrm{~m}^{2}$

- 370 patients with an $\mathrm{eGF}>80 \mathrm{~mL} / \mathrm{min} / 1.73 \mathrm{~m}^{2}$ were prescribed NSAIDs for longer than $48 \mathrm{~h}$.

Conclusions $58 \%$ of the intravenous NSAID prescriptions did not conform to the SPC recommendations. Due to this fact and in order to prevent renal toxicity it is recommended:

1. To establish protocols for pain management during hospitalisation to limit the duration of these drugs to 48 hours and adjust the dose to the patient's renal function.

2. To enhance the proactive role of the pharmacist in individualised patient monitoring.

No conflict of interest.

\section{GRP-072 EXPOSURE TO ANTINEOPLASTIC AGENTS IN ONCOLOGY DEPARTMENTS: PRACTISE SURVEY AND INFORMATION TO THE PERSONNEL OF THREE ONCOLOGY DEPARTMENTS}

doi:10.1136/ejhpharm-2013-000276.072

${ }^{1}$ S Slimano, ${ }^{2} \mathrm{MC}$ Mourad, 'L De Mestier, ${ }^{2} \mathrm{C}$ Regouby, ${ }^{1} \mathrm{O}$ Bouche. ${ }^{1} \mathrm{CHU}$ de Reims, Medicine Ambulatory Unit - Oncology-Hematology, Reims Cedex, France; ${ }^{2} \mathrm{CHU}$ de Reims, Pharmacy, Reims Cedex, France

Background The exposure of pharmacy technicians to antineoplastic agents (AAs) has been widely studied, but less is known about risks of exposure and awareness of nurses, nursing auxiliaries and cleaning personnel.

Purpose To evaluate the practise and the awareness of oncology nurses (ONs), nursing auxiliaries (NAs) and cleaning personnel (CP) concerning exposure to AA.

Materials and Methods Three questionnaires were distributed to ONs, NAs and CP in three oncology wards including one paediatric ward. Participants were asked 10,11 and 12 questions respectively about their practises and awareness of exposure to AAs.

Results For ONs $(n=38)$, gloves are more often worn when manipulating syringes than when manipulating infusion bags $(60.5 \%$ vs. $36.8 \%, p<0.05) .26 .3 \%$ considered themselves well informed but $97.4 \%$ thought information could be improved. $81.6 \%$ of ONs suspected that AAs had teratogenic effects and $10.5 \%$ of them thought that AAs did not have mid- or long-term toxic effects. For NAs $(n=14)$, wearing gloves while washing patients or eliminating excreta was more frequent than mask wearing $(64.3 \%$ vs. $5.3 \%$ ). $28.6 \%$ considered themselves well informed but $92.9 \%$ thought information could be improved. $85.7 \%$ of NAs suspected that AAs had teratogenic effects and $14.3 \%$ of them thought that AAs did not have mid- or long-term toxic effects. For CP $(n=10)$, $62.5 \%$ wore gloves for bed making and $80.0 \%$ for sanitation cleaning. All of them considered themselves not sufficiently informed and $90.0 \%$ thought that AAs had teratogenic effects whereas $10 \%$ of them thought that AAs did not have mid- or long-term toxic effects. All $(n=62)$ reported routine use of water and soap $(46.8 \%)$ or hydro-alcoholic solution (25.8\%) after a potential exposure to AAs. Conclusions Lack of information suggested the necessity of informing the nursing and cleaning personnel on the oncology ward in some fields. A teaching session was arranged by department.

No conflict of interest.

\section{GRP-073 FAILURE MODE AND EFFECT ANALYSIS IN IMPROVING THE SAFETY OF THE CHEMOTHERAPY PROCESS}

doi:10.1136/ejhpharm-2013-000276.073

B Hernandez, C Bravo, MJ Esteban, E García, B Candel, M García, Y Castellanos, J Letellez, J Sierra, FJ Farfán. Hospital de Fuenlabrada, Pharmacy, Madrid, Spain

Background Medication errors in chemotherapy have a high potential to cause harm. Errors may occur during different steps of the medication process.

Failure Mode and Effect Analysis (FMEA) is a proactive risk assessment method that enables potential risks to be identified and prioritises actions to improve safety.

Purpose To apply FMEA methodology to the chemotherapy process: prescribing, pharmaceutical validation, compounding and dispensing.

Materials and Methods Prospective study, in a tertiary level hospital, using the FMEA technique developed by the Veterans Affairs Healthcare System for the chemotherapy process. An interdisciplinary working group was created and meetings held over three months. Processes and subprocesses were described; potential failure modes and possible causes were identified. Main sources used were brainstorming and cause-effect-diagramming. For each failure 